\title{
Performance analysis of an adaptive optics based optical feeder link ground station
}

Nicola Vedrenne, Cyril Petit, A. Montmerle-Bonnefois, C. Lim, J.-M. Conan, et al.

Nicola Vedrenne, Cyril Petit, A. Montmerle-Bonnefois, C. B. Lim, J.-M. Conan, L. Paillier, M.-T. Velluet, K. Caillault, F. Gustave, A. Durecu, V. Michau, F. Cassaing, S. Meimon, J. Montri, "Performance analysis of an adaptive optics based optical feeder link ground station," Proc. SPIE 11852, International Conference on Space Optics - ICSO 2020, 1185219 (11 June 2021); doi: $10.1117 / 12.2599232$ SPIE. Event: International Conference on Space Optics - ICSO 2021, 2021, Online 


\section{International Conference on Space Optics-ICSO 2020}

Virtual Conference

30 March-2 April 2021

Edited by Bruno Cugny, Zoran Sodnik, and Nikos Karafolas
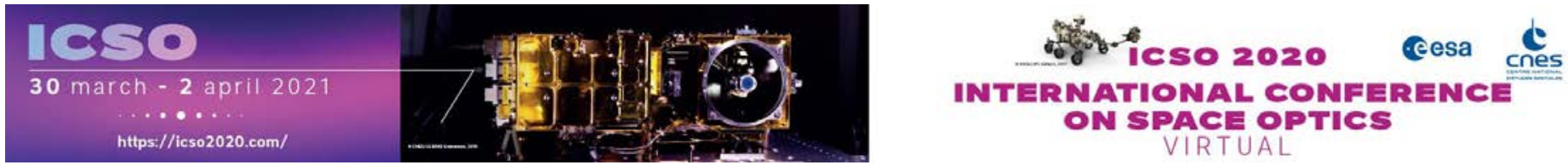

\section{Performance analysis of an adaptive optics based optical feeder link ground station}

\section{Cesa isoporecestings denes}




\title{
Performance analysis of an adaptive optics based optical feeder link ground station
}

\author{
N. Védrenne ${ }^{* 1}$, C. Petit, A. Montmerle-Bonnefois, C. B. Lim, J.-M. Conan, L. Paillier, M.-T. \\ Velluet, K. Caillault, F. Gustave, A. Durecu, V. Michau, F. Cassaing, S. Meimon, J. Montri \\ ONERA, DOTA, Paris Saclay University, F-92322 Châtillon, France
}

\begin{abstract}
Space data highway networks are currently being deployed to provide high data rate connectivity to non-fibre-connected areas around the world and to prepare the incoming growth of Internet Of Things market. Among other solutions, GEO optical feeder links are seen as the backbone of the future globalized very high data rate satellite-based telecommunication networks. The FEEDELIO experiment developed for ESA in April 2019 demonstrated the relevance of angular decorrelation models to assess the statistical characteristics of the pre-compensated uplink irradiance that is deeply affected by anisoplanatism. Based on the conclusion drawn from this experiment, we present the results of a preliminary design study of an Adaptive Optics (AO) pre-compensated optical feeder links ground station. Exploiting end-to-end simulations under relevant turbulence conditions, time-correlated fading statistics are investigated to provide typical fading durations for AO pre-compensated optical channel, taking into account the impact of point-ahead mispointing errors.
\end{abstract}

Keywords: Adaptive optics, optical feeder links pre-compensation.

\section{INTRODUCTION}

To answer the growing need for global connectivity that is currently fueled by the expansion of Internet of Things market and highly connected new emerging working habits, the development of fully optical space data networks [1] will rely on the possibility to bring to space Terabytes of data at the speed of light. This capacity will require very high capacity optical uplinks toward GEO satellites able to reach several tens of Gbps per channel. One of the major challenges for the development of such a capacity is to guarantee link availability despite the dramatic impact of the atmosphere. Cloud masking will be managed thanks to site diversity and the ability to anticipate clouds related interruptions by appropriate handover protocols [2]. Dealing with atmospheric turbulence, it jeopardizes fiber injection efficiency required to reap the benefits of fibered optical amplifiers for the downlink, on the uplink it introduces beam wander and beam spreading. For more than 30 years [3] adaptive optics has been considered as one of the key enabling technologies to make high data rate ground-satellite a reality. On the downlink several demonstrations including longterm exploitation [4] have demonstrated that a commercial exploitation is already possible. For the uplink point ahead anisoplanatism compromises the link performance [5][6]. Feeder link ground station projects are now under development with tradeoffs to be made on baseline architectures and key performance drivers [7]. Among others the selection of the emitter diameter for the uplink has to be performed taking into account the most critical phenomena that could degrade the expected performance. We provide here elements of performance analysis to enlighten the selection of uplink Optical Ground Station (OGS) architectures. Section 2 is dedicated to the presentation of the link budget that is expected from such an OGS. Typical Received Optical Power (ROP) statistics obtained by numerical simulations are provided in section 3.

${ }^{1}$ Nicolas.vedrenne@onera.fr; phone : +33146734757; www.onera.fr 


\section{PRE-COMPENSATED FEEDER LINK BUDGET}

\subsection{Adaptive optics based ground station challenges}

The very basic principle of adaptive optics pre-compensation relies on the ability to probe the downlink turbulence to assess phase-induced perturbations and provide a strictly opposite correction to anticipate the phase aberrations encountered on the uplink. The basic principle of AO based pre-compensation is reminded Figure 1.

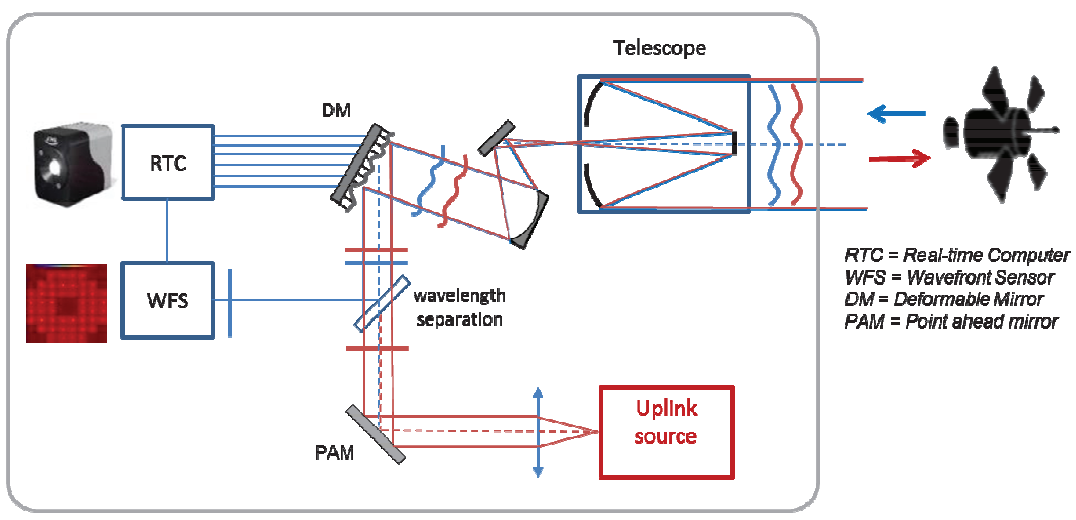

Figure 1 - Adaptive optics based uplink pre-compensation principle.

To compensate for the displacement of both ground station and GEO satellite during the time of flight the uplink beacon is emitted with a slight offset (so called Point Ahead Angle (PAA)) which will be taken equal to 18,5 $\mu$ rad in the following. Due to this PAA, uplink perturbations encountered by the uplink beam are slightly different from the downlink ones used to probe the atmosphere. This phenomenon, called point ahead anisoplanatism, and its consequences on the first moments of uplink ROP have been extensively documented [3][5][6]. The feasibility of uplink precompensation by adaptive optics in a relevant environment has also already been demonstrated [8]. Key challenges remaining to be addressed prior to an on sky demonstration of an uplink high data rate with respect to the ground segment are to:

- Demonstrate the feasibility of high performance, high precision absolute pointing in the direction of the PAA,

- Demonstrate the compatibility of high performance aberration measurements on the downlink considering the power ratio between uplink and downlink;

- Manage the wide variability of environmental conditions required to ensure the availability expected from a high data rate infrastructure.

In this paper we put the stress on the first point so that to investigate the requirements imposed by the expected statistical characteristics of the ROP on the precision of the absolute pointing in the direction of the PAA.

\subsection{Turbulence and wind profiles characteristics}

In order to properly assess point-ahead anisoplanatism influence, it is of critical importance to consider representative turbulence conditions along the line of sight. However, there is little information available on the vertical turbulence statistics at any point of the globe, and this is particularly true for ground station located at sites where turbulence has not been a significant driver so far [2]. As a temporary answer to this need, we have developed a hybrid database of atmospheric turbulence profiles by aggregating vertical turbulence profiles from the opened literature gathered by instrumental measurements in Paranal, Chile [9] and ground $\mathrm{C}_{\mathrm{n}}{ }^{2}$ measurements collected on a 4-month basis in Tenerife [10]. The vertical $\mathrm{C}_{\mathrm{n}}{ }^{2}$ database provides the high altitude layers diversity expected to cover a large scope of anisoplanatism conditions in the free atmosphere, whereas the ground $\mathrm{C}_{\mathrm{n}}{ }^{2}$ measurements provide the ground turbulence variability induced by ground proximity. The link between ground layer and free atmosphere is described thanks to Monin Obukhov Similitude (MOS) theory assuming a $\mathrm{h}^{-\mathrm{p}}$ decrease of the $\mathrm{C}_{\mathrm{n}}{ }^{2}$ with $\mathrm{p}=2 / 3$ for stable conditions (applied for every nighttime ground $\mathrm{C}_{\mathrm{n}}{ }^{2}$ measurements) and with $\mathrm{p}=4 / 3$ for unstable conditions (applied for every daytime ground 
$\mathrm{C}_{\mathrm{n}}{ }^{2}$ measurements). The MOSPAR (for MOS-Paranal) $\mathrm{C}_{\mathrm{n}}{ }^{2}$ database thus built contains more than 10000 different profiles with different integrated parameters.

A selection of specific profiles in this database has been performed on the basis of the most relevant parameters with respect to AO performance. The most significant error terms of a system pre-compensated by AO directly depends on the Fried parameter $\left(\mathrm{r}_{0}\right)$ for the fitting, temporal and aliasing error term, while the anisoplanatism error term is also related to the anisoplanatic angle $\left(\theta_{0}\right)$. Hence, we have selected three different profiles corresponding to different joint cumulative probabilities of occurrence for these two parameters:

- a weak turbulence profile MOSPAR5050 (50\% cumulative joint probability for $\mathrm{r}_{0}$ and $\theta_{0}: 50 \%$ of the profiles of the database present a greater $r_{0}$ and a smaller $\theta_{0}$ than the one of the weak turbulence profile),

- a medium turbulence profile MOSPAR7575 (75\% cumulative joint probability for $\mathrm{r}_{0}$ and $\theta_{0}$ ),

- a strong turbulence profile MOSPAR9090 ( $90 \%$ cumulative joint probability for $\mathrm{r}_{0}$ and $\left.\theta_{0}\right)$.

The integrated turbulence parameters (Fried parameter $\mathrm{r}_{0}$, isoplanatic angle $\theta_{0}$ and log-amplitude variance $\sigma_{\chi}^{2}$ ) of these profiles are provided in Table 1 for a $30^{\circ}$ line of sight at $1.55 \mu \mathrm{m}$. Turbulence distribution along the line of sight is plotted in Figure 2.

\begin{tabular}{|c|c|c|c|}
\hline Profile & $\mathbf{r}_{\mathbf{0}}$ & $\boldsymbol{\theta}_{\mathbf{0}}$ & $\boldsymbol{\sigma}_{\chi}{ }^{2}$ \\
\hline MOSPAR5050 & $7.8 \mathrm{~cm}$ & $11.3 \mu \mathrm{rad}$ & 0.035 \\
\hline MOSPAR7575 & $5.4 \mathrm{~cm}$ & $8.5 \mu \mathrm{rad}$ & 0.048 \\
\hline MOSPAR9090 & $4 \mathrm{~cm}$ & $6.8 \mu \mathrm{rad}$ & 0.079 \\
\hline
\end{tabular}

Table 1 - Integrated turbulence parameters for the three selected turbulence profiles of the hybrid MOSPAR database.

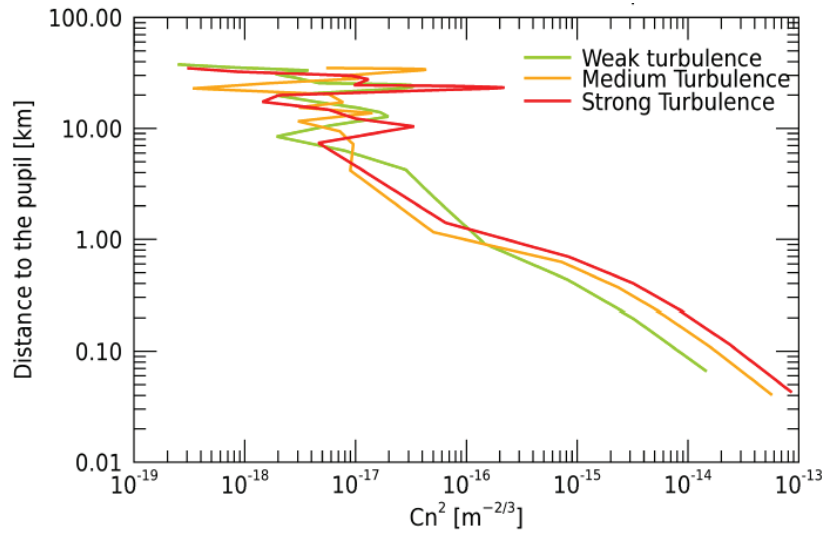

Figure 2 - ONERA's hybrid reference MOSPAR profiles.

The analysis performed in the following sections has been restricted to the most challenging strong turbulence MOSPAR9090 profile.

Considering the wind impact, a Bufton profile [11] has been selected with a $10 \mathrm{~m} / \mathrm{s}$ ground velocity parameter and a 25 $\mathrm{m} / \mathrm{s}$ free atmosphere wind velocity.

\subsection{Typical link budget}

A typical link budget for a 25 Gbps DPSK uplink is synthesized in Table 2. For the space segment we have considered a $25 \mathrm{~cm}$ receiver diameter [12] with a $25 \%$ occultation in diameter and $-5.2 \mathrm{~dB}$ internal losses taking into account optical transmission, coupling losses into a single mode fiber and power allocations to other satellite's function to ensure the optical link (pointing, acquisition and tracking, and optical aberrations including residual mispointing). 
The distance to the satellite is $38609 \mathrm{~km}$ and the elevation is supposed to be $30^{\circ}$. The wavelength considered for the link budget is $1546 \mathrm{~nm}$. The sensitivity of the detector assumed here is taken from experimental measurements on a representative breadboard [13] for a $10^{-3}$ uncoded bit error ratio (BER) with a 25 Gbps DPSK modulation while taking into account the digital processor and transceiver influence (see Blue column of Table 2 in [13]). As stated in [13] an additional sensitivity gain of $1.5 \mathrm{~dB}$, expected from upgrading the digital processor and its connections to other parts of the detection chain is incorporated in the link budget.

The turbulence channel attenuation is obtained from end-to-end simulations using a split-step algorithm, ONERA's TURANDOT code[14], that emulates uplink propagation. In order to simulate AO impact, the phase perturbations are estimated on a downlink plane wave sharing the same aperture as the uplink beam. A perfect phase pre-compensation is therefore applied to the uplink beam which undergoes the same turbulence occurrence. PAA impact is taken into account by an appropriate shift of the phase screens located in altitude. The influence of an uplink mispointing can also be considered by adding a tip/tilt offset to the emitted optical wave before uplink propagation.

In order to reap the benefit of a large transmitter gain, $\mathrm{D}=0.6 \mathrm{~m}$ diameter $(\lambda / \mathrm{D}=2.6 \mu$ rad diffraction limit) is considered as a baseline for the OGS, with a $30 \%$ central occultation in diameter. To alleviate the impact of anisoplanatism error (increasing with the diameter), a less ambitious $\mathrm{D}=0.2 \mathrm{~m}(\lambda / \mathrm{D}=7.7 \mu \mathrm{rad}$ diffraction limit) is also considered. Concerning the uplink source, recent developments on high power, high stability and low distortion fiber laser sources [15] make it credible that a $50 \mathrm{~W}$ source compatible with DPSK modulation will be available within the coming months as such a source is currently under development at ONERA.

For the atmospheric transmission contributor, cumulative optical transmission statistical analysis has been performed based on 4 years of data, between 2015 and 2018 for the city of Nice (South of France). Physical parameters necessary to compute cloud optical depth (liquid and ice water content) are extracted from ERA5 reanalysis (temporal resolution $1 \mathrm{~h}$, spatial resolution $0.25^{\circ}$ ). Aerosol data come from Copernicus Atmosphere Monitoring Service analyses (temporal resolution $3 \mathrm{~h}$, spatial resolution $0.8^{\circ}$ ), aerosols optical depth at $1240 \mathrm{~nm}$ is used [16]. The wavelength is supposed to be outside of atmosphere absorption peaks. This analysis leads to allocate $-3.8 \mathrm{~dB}$ to thin clouds atmospheric transmission and $-0.2 \mathrm{~dB}$ to aerosols for a zenithal line of sight, hence leading to a total of $-8 \mathrm{~dB}$ for a $30^{\circ}$ elevation. This allocation corresponds to a $90 \%$ cumulative probability of having an atmospheric transmission more favorable than $-8 \mathrm{~dB}$ assuming a cloud-free line of sight, and an overall link availability of $58 \%$ of the time, the line of sight being totally occulted by clouds $36 \%$ percent of the time.

The figures presented in Table 2 are obtained without any calibration error.

\begin{tabular}{|c|c|c|c|c|}
\hline \multicolumn{2}{|r|}{ Name } & unit & $\mathrm{D}=0.2 \mathrm{~m}$ & $D=0.6 \mathrm{~m}$ \\
\hline \multirow{4}{*}{ OGS } & Output power HPOA average & $\mathrm{dBm}$ & \multicolumn{2}{|c|}{47,0} \\
\hline & Tx Optical Antenna Gain & $\mathrm{dB}$ & 109,9 & 119,5 \\
\hline & Tx Optical Transmission & $\mathrm{dB}$ & \multicolumn{2}{|c|}{$-2,2$} \\
\hline & Tx Static aberration losses & $\mathrm{dB}$ & \multicolumn{2}{|c|}{$-0,5$} \\
\hline \multirow{2}{*}{ Channel } & Loss clouds and aerosols & $\mathrm{dB}$ & \multicolumn{2}{|c|}{$-8,0$} \\
\hline & Loss free space propagation & $\mathrm{dB}$ & \multicolumn{2}{|c|}{$-289,9$} \\
\hline \multirow{2}{*}{ SAT } & Rx Optical Antenna Gain & $\mathrm{dB}$ & \multicolumn{2}{|c|}{114,1} \\
\hline & Rx Internal losses & $\mathrm{dB}$ & \multicolumn{2}{|c|}{$-5,2$} \\
\hline \multicolumn{2}{|c|}{ TOTAL without turbulence uplink } & $\mathrm{dBm}$ & $-34,8$ & $-25,3$ \\
\hline \multirow{2}{*}{$\begin{array}{l}\text { OGS \& } \\
\text { Channel }\end{array}$} & $\begin{array}{l}\text { Turbulence induced losses } \\
\text { (mean signal, no mispointing } \\
\text { error) }\end{array}$ & $\mathrm{dB}$ & $-0,2$ & $-3,0$ \\
\hline & $\begin{array}{l}\text { Residual fluctuations margin } \\
@ 99 \% \text { (Full AO) }\end{array}$ & $d B$ & $-3,0$ & $-9,8$ \\
\hline \multicolumn{2}{|c|}{ Mean received optical power } & $\mathrm{dBm}$ & $-38,0$ & $-38,1$ \\
\hline \multicolumn{2}{|c|}{$\begin{array}{l}\text { Required Received optical power @25 Gb/s, } \\
\text { BER = 10-3,DPSK (DPU+transceiver) }\end{array}$} & $\mathrm{dBm}$ & \multicolumn{2}{|c|}{$-41,2$} \\
\hline \multicolumn{2}{|c|}{ Additional sensitivity upgrade (DPU) } & $d B$ & \multicolumn{2}{|c|}{$-1,5$} \\
\hline \multicolumn{2}{|l|}{ Link margin } & $\mathrm{dB}$ & \multicolumn{2}{|c|}{3,0} \\
\hline \multicolumn{2}{|c|}{ Required sensitivity } & $\mathrm{dBm}$ & \multicolumn{2}{|c|}{$-39,7$} \\
\hline \multicolumn{2}{|c|}{\begin{tabular}{|l} 
Additionnal link margin \\
\end{tabular}} & $\mathrm{dB}$ & 1,7 & 1,6 \\
\hline
\end{tabular}

Table 2 - Uplink link budget (left for a $0.2 \mathrm{~m}$ unocculted aperture, right for a $\mathbf{0 . 6} \mathbf{m}$ occulted aperture).

As shown by the table, a limited $1.6 \mathrm{~dB}$ (respectively $1.7 \mathrm{~dB}$ ) margin can be expected if no mispointing error is introduced by the PAA application. 
However, as it is illustrated in Figure 1, the PAA is introduced via the point-ahead mirror (PAM) which is controlled in open-loop. The precision of PAA application will therefore depend on the capacity to accurately quantify the magnification of the optical train between the output of the uplink source and the emitting aperture of the telescope and to ensure that the appropriate angle is actually applied given the stability and the accuracy of the PAM. Several possibilities can be considered for doing so: exploiting the optical signal of calibrated bright binary stars, optimizing the averaged uplink signal detected by the satellite... In order to appropriately design the on-sky calibration procedure of the PAM actuation we investigate in the following section the impact of a PAA calibration error on the turbulence channel statistical characteristics.

\section{PERFORMANCE ANALYSIS}

\subsection{Numerical simulation results in a phase conjugation approach}

The impact of calibration errors of the point-ahead angle is assessed here by numerical simulations. A thousand independent occurrences of uplink pre-compensated channel ROP are generated and analyzed statistically. Results are reported in Figure 3 for the two diameters configurations (left for $\mathrm{D}=0.2 \mathrm{~m}$ unocculted, right for $\mathrm{D}=0.6 \mathrm{~m}$ with $30 \%$ occultation in diameter) in red for the PAA direction. To illustrate the detrimental impact of point-ahead anisoplanatism the ROP at the focal plane of the satellite is also considered without PAA in black. For each case three quantities are considered:

- the average ROP upon the overall statistics (continuous line),

- the $99 \%$ cumulative probability threshold corresponding to the attenuation for which the signal is above this threshold with a $99 \%$ probability (dashed line),

- the maximum encountered value (mixed line).

A first analysis of the figure confirms the appropriateness of the $0.2 \mathrm{~m}$ case to benefit for a configuration robust to mispointing errors and point ahead anisoplanatism: no significant difference is observed without and with PAA, and only a slight modification of the average flux and of the $99 \%$ threshold is observed (less than $0.5 \mathrm{~dB}$ ) when increasing the mispointing error.

The second observation that can be drawn is the significant dynamic of ROP fluctuations for this $20 \mathrm{~cm}$ case: $7 \mathrm{~dB}$ between max and 99\% threshold whatever the configuration (with or without PAA, with or without mispointing error). Theses fluctuations are directly caused by diffractive effects of the propagation: they are not corrected by AO, AO being a phase only correction method. These diffractive effects, responsible for downlink fluctuations of the collected flux in the receiver aperture, also affect the uplink beam. This effect will be all the more significant that the considered diameter is small compared to the typical size of the downlink speckle. In our case the typical speckle size can be approximated by the Fresnel length $\sqrt{ } \lambda \mathrm{L}$, L being the overall propagation distance through the atmosphere, and we find $\sqrt{ } \lambda \mathrm{L}=21 \mathrm{~cm}$ for $\mathrm{L}$ $=30 \mathrm{~km}$, which is very close to the transmitter diameter, hence the significant power fluctuations encountered on the uplink pre-compensated beam.

Anisoplanatism is significant for the $\mathrm{D}=0.6$ diameter. Without mispointing error, a $3 \mathrm{~dB}$ average loss appears between the case without PAA and the case with PAA. Whereas an $8 \mathrm{~dB}$ gain is expected from the increased diameter (geometrical gain provided by the smaller diffraction spot of the $\mathrm{D}=0.6 \mathrm{~m}$ diameter including central occultation influence) this expected gain is limited to $6.5 \mathrm{~dB}$ due to anisoplanatism.

Whereas aperture averaged scintillation influence is significantly reduced comparing to the $\mathrm{D}=0.2 \mathrm{~m}$ case, as it is illustrated by the very limited dynamic of ROP fluctuations when no anisoplanatism is introduced, anisoplanatism is responsible for very significant variations of the ROP: close to $13 \mathrm{~dB}$ without mispointing error, even more if a mispointing error is introduced. This can be explained by the specific structure of anisoplanatism residuals. Anisoplanatism is particularly significant on the tip/tilt modes whereas the diffraction pattern in the satellite plane remains almost not affected by higher order terms which are corrected by AO: we are in the typical case of a diffraction spot randomly displaced in front of a pin-hole (the satellite), but slightly decentered (due to mispointing). Due to mispointing the position of diffraction pattern is wandering in the satellite plane and the satellite pupil might be rarely located on the steep edge of the diffraction pattern, hence causing rare but strong power fluctuations.

Considering the overall link budget of Table 2, the power loss allocation to mispointing error must be limited to $1.6 \mathrm{~dB}$. According to Figure 3, this leads to a maximum mispointing error of $0.2 \mu \mathrm{rad}$ (more than 10 times smaller than the 
diffraction limit), which is particularly challenging but should remain manageable assuming an adequate calibration process and PAM control.
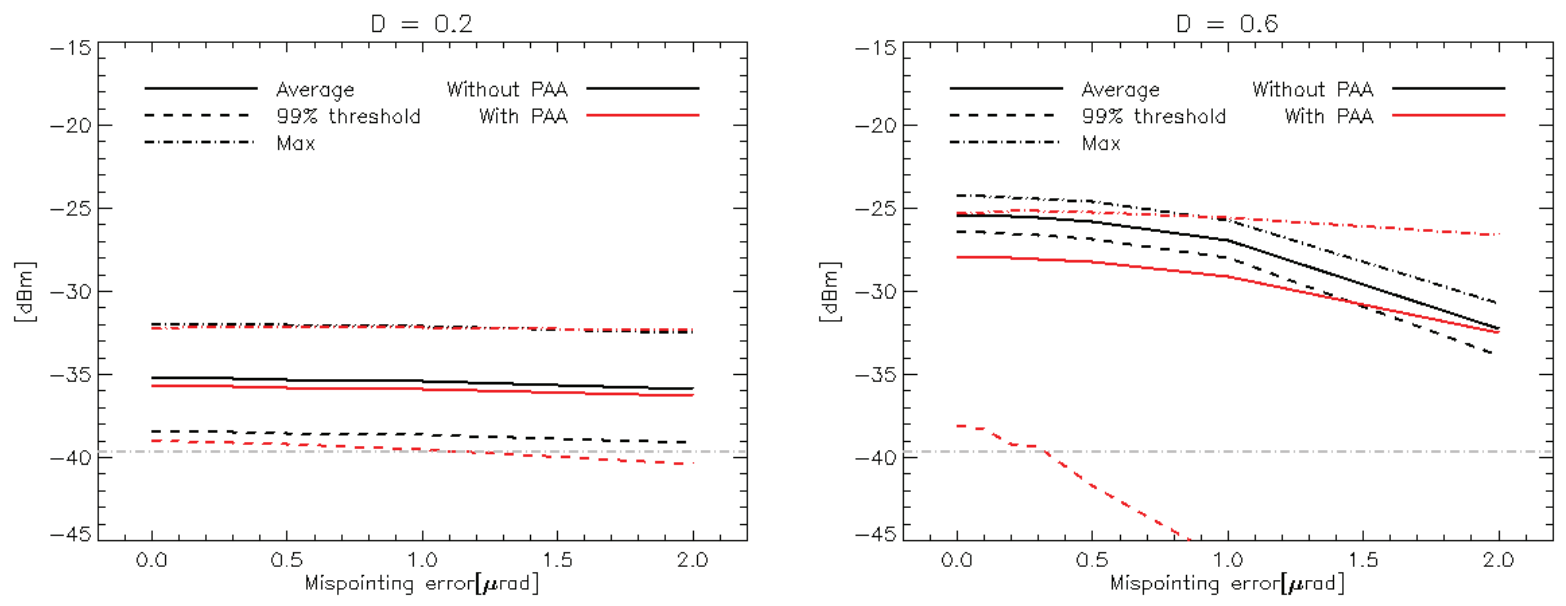

Figure 3 - Impact of miscalibration error (mispointing) of the PAA for the two uplink configurations.

\subsection{Temporal characteristics}

In this section we compare the temporal characteristics of typical time series of ROP (4.7 khz sampling) affected by point ahead anisoplanatism and a $0.2 \mu \mathrm{rad}$ mispointing error for the two considered diameters: Figure 4 for the $0.2 \mathrm{~m}$ diameter and Figure 5 for the $0.6 \mathrm{~m}$ diameter. The time series has been generated thanks to temporally correlated simulations. AO correction is still assumed to be perfect (phase conjugation case). The raw ROP time series are plotted in black. Both time series shows very different temporal characteristics. The $0.2 \mathrm{~m}$ case shows numerous (typ. 30 occurrence), short (typ. $5 \mathrm{~ms}$ ) and limited amplitude fadings (typ. $3 \mathrm{~dB}$ deepness) whereas the $0.6 \mathrm{~m}$ show a limited number (typ. 10) of very deep fadings (up to $12 \mathrm{~dB}$ attenuation).

In order to push forward the comparison between the two emitter diameters, we investigate the effect of an increasing interleaving window on the simulated time-series. Our approach consists here to convolve anisoplanatism induced ROP time series by a constant and convolutive kernel of increasing length and unitary norm hence performing a local temporal average by a moving window. The objective of this approach is to assess the ability of an interleaving process to cope with the encountered fading temporal characteristics. This approach assumes that the synchronization process and the amplitude of the signal can be properly managed by the detection, synchronization and demodulation chain and neglects the impact of the different noise sources on the efficiency of the interleaving process. This is, somewhat, a very optimistic assumption only dedicated to the study of what the typical size of an interleaving window would be to reduce the fading impact. The impact of a $10 \mathrm{~ms}$ convolution window is plotted in purple, $20 \mathrm{~ms}$ window is plotted in red and 50 $\mathrm{ms}$ in brown. For $\mathrm{D}=0.2 \mathrm{~m}$ a $10 \mathrm{~ms}$ convolution reduces the deepest fading amplitude of $3 \mathrm{~dB}$. For $\mathrm{D}=0.6 \mathrm{~m}$, a $20 \mathrm{~ms}$ window reduces the deepest fading amplitude of $9 \mathrm{~dB}$. Considering the same $20 \mathrm{~ms}$ convolution window, a $5 \mathrm{~dB}$ additional margin is obtained with the $\mathrm{D}=0.6 \mathrm{~m}$ compared to the $\mathrm{D}=0.2 \mathrm{~m}$ case.

We illustrate here the critical importance of the development of appropriate interleaving process to cope with uplink remaining ROP fluctuations and the potential gain that can be expected from the use of a large uplink diameter, assuming such a large amplitude detection, digitization, and data processing chain remains feasible. 


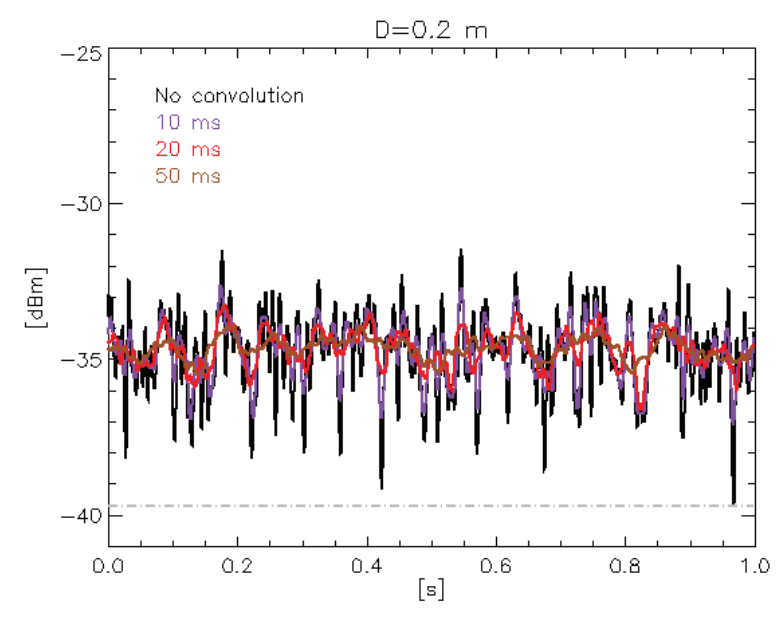

Figure 4 - Received optical power time series in the satellite plane convolved with constant convolution kernel of different sizes for the $\mathbf{0 . 2} \mathbf{m}$ uplink diameter.

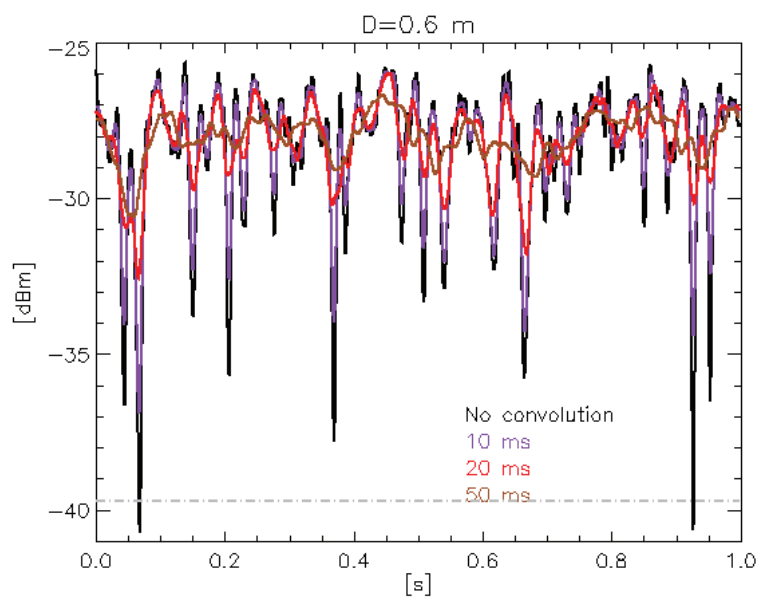

Figure 5 - Received optical power time series in the satellite plane convolved with constant convolution kernel of different sizes for the $\mathbf{0 . 6} \mathbf{~ m}$ uplink diameter.

\section{CONCLUSION}

We have presented a performance analysis of an uplink AO pre-compensated OGS architecture. Two configurations were considered: a $0.2 \mathrm{~m}$ unocculted aperture and a $0.6 \mathrm{~m}$ aperture with $30 \%$ central occultation. Atmospheric turbulence and transmission characteristics were chosen to provide a performance evaluation withstanding propagation conditions encountered more than $90 \%$ of the time (assuming a cloud-free line of sight). In both cases a link budget has been provided taking into account fading statistics in the presence of point-ahead anisoplanatism. Both configurations enable to close the link budget. The $0.6 \mathrm{~m}$ case is particularly demanding in terms of PAA calibration precision as a $0.2 \mu \mathrm{rad}$ mispointing error adds a more than $1 \mathrm{~dB}$ penalty to the $99 \%$ cumulative probability threshold. However, it enables to expect a $5 \mathrm{~dB}$ gain with respect to the $0.2 \mathrm{~m}$ case assuming the use of $20 \mathrm{~ms}$ (or longer) interleavers. These $5 \mathrm{~dB}$ additional gain provides an additional margin to address even more demanding data rates.

Results presented here were obtained considering a perfect $\mathrm{AO}$ and taking into account anisoplanatism, but neglecting other usual AO error budget terms (both spatial and temporal error terms), anisoplanatism being the performance killer. To consolidate these results, a more realistic AO correction will be simulated. Nevertheless, considering state of the art components and already demonstrated performance in a relevant environment [8], expected results shall not significantly deviate from those presented here.

Building on these results ONERA is currently designing an AO system dedicated to uplink pre-compensation for GEO feeder links. The FELIN-AO (Feeder LINk adaptive optics) bench will be able to address both uplink configurations ( $0.2 \mathrm{~m}$ and $0.6 \mathrm{~m}$ with occultation). This $\mathrm{AO}$ bench will be tested in a relevant environment within the Vertigo project outdoor demonstration [17].

\section{AKNOWLEDGEMENT}

The authors wish to thank J. Osborn for kindly providing Paranal's $\mathrm{C}_{\mathrm{n}}^{2}$ profiles database.

\section{REFERENCES}

[1] H. Hauschildt et al., "HydRON: High thRoughput Optical Network," in 2019 IEEE International Conference on Space Optical Systems and Applications (ICSOS), Oct. 2019, pp. 1-6, doi: 10.1109/ICSOS45490.2019.8978985.

[2] S. Poulenard, M. Crosnier, and A. Rissons, "Ground Segment Design for Broadband Geostationary Satellite With Optical Feeder Link,” J. Opt. Commun. Netw., JOCN, vol. 7, no. 4, pp. 325-336, Apr. 2015. 
[3] R. K. Tyson, “Adaptive optics and ground-to-space laser communications,” Appl. Opt., AO, vol. 35, no. 19, pp. 3640-3646, Jul. 1996, doi: 10.1364/AO.35.003640.

[4] Karen Saucke, Christoph Seiter, Frank Heine, Mark Gregory, Daniel Tröndle, Edgar Fischer, Thomas Berkefeld, Mikael Feriencik, Marco Feriencik, Ines Richter, Rolf Meyer, "The Tesat transportable adaptive optical ground station," Proc. SPIE 9739, Free-Space Laser Communication and Atmospheric Propagation XXVIII, 973906 (15 March 2016); https://doi.org/10.1117/12.2218275

[5] N. Védrenne, J. Conan, A. Bonnefois, C. Petit, M. Velluet, and V. Michau, "Adaptive optics pre-compensation for GEO feeder links: Towards an experimental demonstration," in 2017 IEEE International Conference on Space Optical Systems and Applications (ICSOS), Nov. 2017, pp. 77-81, doi: 10.1109/ICSOS.2017.8357215.

[6] J.-M. Conan, A. Montmerle-Bonnefois, N. Védrenne, C.B. Lim, C. Petit, V. Michau, M.-T. Velluet, J.-F. Sauvage, S. Meimon, C. Robert, J. Montri, F. Mendez, P. Perrault, G. Artaud, B. Benammar, « Adaptive Optics for GEO-Feeder Links: from performance analysis via reciprocity based models to experimental demonstration », COAT-2019 - workshop, ONERA, Dec 2019, Châtillon, France, https://doi.org/ 10.34693/COAT2019-S5-003

[7] M. Toyoshima et al., "Research and development on a hybrid high throughput satellite with an optical feeder link - Study of a link budget analysis," in 2017 IEEE International Conference on Space Optical Systems and Applications (ICSOS), Nov. 2017, pp. 267-271, doi: 10.1109/ICSOS.2017.8357424.

[8] N. Védrenne et al., "First Experimental Demonstration of Adaptive Optics Pre-Compensation for GEO Feeder Links in a Relevant Environment," in 2019 IEEE International Conference on Space Optical Systems and Applications (ICSOS), Oct. 2019, pp. 1-5, doi: 10.1109/ICSOS45490.2019.8978970.

[9] J. Osborn et al., "Optical turbulence profiling with Stereo-SCIDAR for VLT and ELT," Monthly Notices of the Royal Astronomical Society, vol. 478, no. 1, pp. 825-834, Jul. 2018, doi: 10.1093/mnras/sty1070.

[10]D. Sprung and E. Sucher, "Characterization of optical turbulence at the solar observatory at the Mount Teide, Tenerife," in Remote Sensing of Clouds and the Atmosphere XVIII; and Optics in Atmospheric Propagation and Adaptive Systems XVI, Oct. 2013, vol. 8890, p. 889015, doi: $10.1117 / 12.2032744$.

[11]L. C. Roberts et al., "Improved models of upper-level wind for several astronomical observatories," Opt. Express, vol. 19, no. 2, 2011.

[12] B. Roy et al., "Optical feeder links for high throughput satellites," in 2015 IEEE International Conference on Space Optical Systems and Applications (ICSOS), New Orleans, LA, USA, Oct. 2015, pp. 1-6, doi: 10.1109/ICSOS.2015.7425074.

[13]T. Anfray et al., "Assessment of the Performance of DPSK and OOK Modulations at 25 Gb/s for SatelliteBased Optical Communications," in 2019 IEEE International Conference on Space Optical Systems and Applications (ICSOS), Oct. 2019, pp. 1-6, doi: 10.1109/ICSOS45490.2019.8978982.

[14] N. Védrenne et al., "Turbulence effects on bi-directional ground-to-satellite laser communication systems," Proc. International Conference on Space Optical Systems and Applications (ICSOS) 2012, 10-1, Corsica, France, October 9-12 (2012).

[15]P. Kaczmarek, D. Stachowiak, and K. M. Abramski, "40 W All-Fiber Er/Yb MOPA System Using SelfFabricated High-Power Passive Fiber Components," Applied Sciences, vol. 8, no. 6, Art. no. 6, Jun. 2018, doi: 10.3390/app8060869.

[16] Morcrette, and Coauthors, 2009: Aerosol analysis and forecast in the ECMWF Integrated Forecast System. Part I: Forward modelling, J. Geophys. Res., 114, D06206, https://doi.org/10.1029/2008JD011235.

[17]Le Kernec et al., "The H2020 VERTIGO Project : Towards Tbit/s Optical Feeder Links," ICSO conference (2021) 